\title{
Emergent Axions
}

\section{P. Anastasopoulos ${ }^{* 1}$, P. Betzios ${ }^{2}$, M. Bianchi ${ }^{3}$, D. Consoli ${ }^{1}$, E. Kiritsis ${ }^{2,4}$}

${ }^{1}$ Mathematical Physics Group, University of Vienna

Boltzmanngasse 51090 Vienna, Austria.

2 Crete Center for Theoretical Physics

Department of Physics, University of Crete, Herakleion, Greece.

3 Dipartimento di Fisica, Università di Roma "Tor Vergata"

I.N.F.N. Sezione di Roma "Tor Vergata”, Via della Ricerca Scientifica, 00133 Roma, Italy.

${ }^{4}$ APC, Université Paris 7, CNRS/IN2P3, CEA/IRFU, Obs. de Paris, Sorbonne Paris Cité,

Bâtiment Condorcet, F-75205, Paris Cedex 13, France (UMR du CNRS 7164).

This proceeding is based on [1] where we study a model in which the SM is coupled to a Hidden sector via heavy messengers. In this framework, instanton densities of the Hidden sector behave in the IR like axions coupled to the SM. We study the general properties of such emergent axions and we show that they are different from both fundamental and composite axions that have been studied so far.

Corfu Summer Institute 2019 "School and Workshops on Elementary Particle Physics and Gravity" (CORFU2019)

31 August - 25 September 2019

Corfù, Greece

${ }^{*}$ Speaker. 


\section{Introduction}

Gravity poses some of the biggest the biggest puzzle of modern physics. Even though it was the first interaction that was discovered, it still remains the least understood. There are two main reasons for this. First, a quantum field theory (QFT) of gravity suffers from divergences. Second, the cosmological constant problem, aka the value of the energy density of the vacuum has an extremely small value.

Until now, the only theory that gives a consistent description of quantum gravity is string theory, which provides a natural cutoff, the string scale, protecting the theory from divergences. On the other hand, new holographic ideas relate large-N gauge theories to string theories (which contain gravity) providing a different approach to this problem.

Being motivated by paradigms emerging from the AdS/CFT correspondence, E. Kiritsis proposed a completely new approach where Gravity is not a fundamental force [2], but it emerges via a hidden sector which interacts with the Standard Model (SM) via messenger fields. In this framework, we bypass both problems mentioned above. First, Gravity has a natural upper scale avoiding divergences since it is an effective theory, and second the cosmological constant is also emergent and it depends on various scales of this setup, allowing for its small value [3].

Apart from gravity, there are also other operators of the hidden sector that appear as weakly coupled emergent fields to the SM. Of particular interest are instanton densities and currents associated with global symmetries of the hidden sector that appear as emergent axions and emergent gauge fields coupled to the SM [4], [5]. In addition, composite fermions can also emerge from the hidden sector and can play the role of the right handed neutrinos.

In our paper [1], we focus on emergent axions.

\section{The framework}

We follow the framework introduced in [2] where all interactions in nature are described by four-dimensional gauge theories without gravity. In this picture the SM is one part of them while the rest are what we will refer to as "hidden sectors". A crucial assumption is that the number of degrees of freedom of the hidden sectors is much larger than the SM one. We can also consider a simplified version in which there is only one dominant hidden sector with an enormous number $N$ of degrees of freedom compared to the SM.

In order to have an UV complete theory, the hidden sector has to be strongly coupled or conformal. Together with the large- $N$ assumption, this allows a possible holographic description for the hidden sector. In the UV the two sectors are coupled via messengers. The UV completeness enforces messengers to be bifundamental fields charged under both sectors, [2], and heavily massive compared to SM scales. At low energies, integrating out messengers, in the effective action the SM and the hidden theory are coupled by (irrelevant) double (or multiple) trace operators ${ }^{1}$.

From an IR perspective in the SM sector, the hidden sector can manifest its presence via emergent fields, that can play the role of low-energy dynamical fields coupled to the SM. In addition, we only need to include a few number of such emergent fields in the low energy description. For

\footnotetext{
${ }^{1}$ There is an exception, [7], but we shall not consider it in this proceeding.
} 
instance for scalar operators, most of them acquire effective masses of the order of the messenger scale $M$, therefore they are irrelevant for low-energy physics. There are only three classes of operators that are protected by symmetries, and therefore have generically low masses:

- The total conserved stress tensor, which gives rise to emergent gravity, [2, 3]

- Conserved global currents, which give rise to emergent gauge fields, [2, 4]

- Instanton densities, which are protected by their topological invariance and give rise to emergent axions, [1, 2]

The first class is the most intriguing. Emergent gravitons can appear in the low energy theory as a coupling between stress tensors, schematically

$$
S \sim \int d^{4} x \frac{T_{\mu v}^{\mathrm{hidden}}}{M^{4}} T_{\mathrm{SM}}^{\mu v}
$$

where $M$ is the messenger scale and $T_{\mu \nu}^{\text {hidden/SM }}$ are the stress-energy tensors of the two sectors. The hidden stress tensor plays the role of a metric perturbation, $T_{\mu v}^{\text {hidden }} \sim M^{4} h_{\mu v}$. The hidden sector, being a large- $N$ strongly coupled theory, it should generate semiclassical gravity according to the holographic correspondence. A deep analysis for this topic is a work in progress [3]. There might be the possibility of other emergent composite fields such as fermions playing the role of righthanded neutrinos. As we have already mentioned, in [1] we focus on the third class of fields, the emergent axions.

Axions were introduced in the work of Peccei and Quinn [6] in order to solve the strong CPproblem in QCD. In this work the theory was not renormalizable because of the axion coupling to the QCD-instanton density [8, 9]. Soon after renormalizable theories of axions were constructed [10]-[13] and axion couplings to matter have been determined [14]-[19]. The role of axions has expanded beyond QCD, as they become candidates for the dark matter and inflatons, [20]-[26].

From an effective field theory point of view, axions are a special class of fields. They are (pseudo-)scalar fields that possess a (perturbative) shift symmetry and couple to instanton densities. At a non-perturbative level, in all the known cases the shift symmetry is broken at best to a discrete symmetry due to instanton effects. These effects are also responsible for giving a mass and a potential to the axion ${ }^{2}$.

In string theory axions appear in two forms ${ }^{3}$ : as a Ramond-Ramond massless scalar in type IIB string theory or as product of compactification from internal components of antisymmetric form gauge fields and off-diagonal components of the metric. In both cases they are generalized gauge fields of string theory and therefore have accompanying gauge symmetries [29, 30] which provide the perturbative Peccei-Quinn (PQ) symmetries in String theory [31]. Again these symmetries are broken by non-perturbative effects ${ }^{4}$.

In this proceeding, we will discuss a novel type of axion, the composite or emergent axion. The idea of realising the axion as a composite particle is not new, [47]-[55]. In most of these

\footnotetext{
${ }^{2}$ See also for a recent review [18].

${ }^{3}$ See [27] for a review and [28] for an effective theory discussion.

${ }^{4}$ See [32]-[46] for several cases.
} 
realizations axions are 'axi-pions' of a strongly-coupled 'axi-gauge' theory. The characteristic energy scale $f_{a}$ is determined by the strong coupling scale of the axi-gauge theory, $f_{a} \sim \Lambda_{a}$ and to generate a reasonable composite axion one needs $\Lambda_{a} \gg \Lambda_{Q C D}$, [20].

Our main goal in this proceeding is to specifically analyze the case where the hidden theory is a holographic theory, i.e. a large- $N$ strongly coupled gauge theory. As we shall see, such axions are novel, both in terms of their identification (they will correspond to the $0^{+-}$glueball generated by instanton densities operators instead of $\eta^{\prime}$ s) and their properties. Furthermore the emergent axions we discuss do not rely on fermionic PQ symmetries, but rather on the approximate PQ symmetry associated to instanton densities in gauge theories. This symmetry is exact in perturbation theory, but it is violated non-perturbatively by instanton effects. This gives a mass to the axion, similarly to the standard case.

Recently a holographic QCD axion model has been proposed in [21] and provides a different way of realizing a composite axion.

\section{Composite axions}

We would like to compute the direct couplings of the instanton density operator $\operatorname{Tr}[F \wedge F]$ in the hidden large- $N$ sector to instanton density operators of the SM.

The couplings of messengers to the SM fields and to the hidden sector fields can be schematically written as

$$
S_{i n t}=\int d^{4} x\left[\phi_{a i}^{*} \phi_{a j} \chi^{i j}+\phi_{a i}^{*} \phi_{b i} A^{a b}\right]
$$

where we have suppressed derivatives and other space-time indices. All the fields above are written as bifundamentals ${ }^{5}$ and can be vector bosons, fermions or scalars. $\phi_{a i}$ are messenger fields, where the index $a$ is the color index of the hidden sector while $i$ is a gauge index of a SM gauge group. $\chi_{i j}$ and $A_{a b}$ are respectively the SM and hidden sector fields.

The effective coupling between instanton densities of the SM and the hidden sector can easily arise. As an example in a perturbative setup, we consider a four-point one-loop amplitude with two non-abelian gauge fields in hidden sector and two gauge fields on the SM side ${ }^{6}$. Considering for simplicity only fermionic messengers circulating in the loop, one can extract the effective couplings integrating out the messengers obtaining the following effective lagrangian

$$
\begin{aligned}
S_{\mathrm{eff}}=-\frac{g_{h}^{2} g_{S M}^{2}}{90(4 \pi)^{2} M^{4}} \int d^{4} x & {\left[G_{\mu \nu}^{a} G^{a, \mu v} F_{\rho \sigma}^{i} F^{i, \rho \sigma}+\frac{7}{4} G_{\mu \nu}^{a} \widetilde{G}^{a, \mu v} F_{\rho \sigma}^{i} \widetilde{F}^{i, \rho \sigma}+\right.} \\
& \left.+\frac{7}{2} G_{\mu \nu}^{a} \widetilde{G}_{\rho \sigma}^{a} F^{i, \mu v} \widetilde{F}^{i, \rho \sigma}+2 G_{\mu \nu}^{a} G_{\rho \sigma}^{a} F^{i, \mu v} F^{i, \rho \sigma}\right]
\end{aligned}
$$

where $F(G)$ are the field strengths of the gauge bosons in the SM (hidden theory). Similar couplings are also expected with bosonic messengers. This calculation is perturbative, but also indicative of the general case, which is not necessarily perturbative.

\footnotetext{
${ }^{5}$ There are many ways to write the SM fields as bifundamentals. All these ways have been classified in [56], and they involve the inclusion of at least one more and typically several extra U(1)s, which are generically anomalous, [57]-[64].

${ }^{6}$ This class of calculations is in the "light by light scattering" class and various aspects have been addressed in the literature, [65]-[74].
} 
At large $N$ and strong coupling, tensor fields, present in the second line of (3.2), are expected to acquire large anomalous dimensions and are not expected to be important at low energy. As we will show later on, scalar couplings, as the first coupling in the first line in (3.2), are also not very relevant at low energy. It is only the coupling of instanton densities, the second coupling in the first line in (3.2), that turns out to be important in the IR and will give rise to an emergent axion.

\subsection{IR resolution}

We would like to reinterpret the interaction between two scalar operators in the two theories, like the ones shown in the first line of (3.2), in an effective description. To do this, we would like to address the more general problem of a scalar-scalar interaction between two theories and its IR "resolution". We consider two theories coupled via an interaction of the form

$$
S=S_{h}\left[O_{h}\right]+S_{S M}\left[O_{S M}\right]+\lambda \int d^{4} x O_{h}(x) O_{S M}(x)
$$

where $O_{h}\left(O_{S M}\right)$ is an operator of dimension $\Delta_{h}\left(\Delta_{S M}\right)$ belonging to the hidden (SM) sector. If we identify $O_{S M}$ and $O_{h}$ as the instanton densities $\operatorname{Tr}[F \wedge F]$ and $\operatorname{Tr}[G \wedge G]$ respectively in (3.2), then $\Delta_{h}=\Delta_{S M}=4$. Coming back to general scalar operators, the coupling $\lambda$ has a suppression from the messengers scale $M$

$$
\lambda=\lambda_{0} M^{4-\Delta_{h}-\Delta_{S M}},
$$

with $\lambda_{0}$ dimensionless. Normalizing $O_{h}$ in such a way that all its correlators are of order $\mathscr{O}\left(N^{2}\right)$, the coupling $\lambda_{0}$ is of order $\mathscr{O}(1)$. As consequence the higher than three-point (connected) functions are suppressed compared to two-point functions.

We would like now to interpret the presence of the effective interaction $S_{\text {int }}$ in (3.3) from the point of view of the SM as due to a dynamical scalar $a$, coupled linearly to the operator $O_{S M}$. The effective action reads

$$
S_{e f f}=\int \frac{d^{4} p}{(2 \pi)^{4}}\left[a(p) K_{a}(p) a(-p)+g a(p) O_{S M}(-p)\right]+S_{S M}
$$

where $K_{a}$ is a kinetic function to be determined. We are choosing the axion field to be dimensionless, therefore comparing the effective action with (3.3) we get $a \sim M^{-\Delta_{h}} O_{h}$ and $g \sim M^{4-\Delta_{S M}}$. The axion mass $m_{a}$ and the axion decay constant $f_{a}$ can be extracted from the IR expansion of the two-point correlator function of $a$

$$
i\langle a(p) a(-p)\rangle^{-1} \simeq f_{a}^{2}\left(p^{2}+m_{a}^{2}\right)+\mathscr{O}\left(p^{4}\right)
$$

The correlator can be obtained from the effective action (3.5), we get [1]

$$
-i\langle a(p) a(-p)\rangle=\frac{-i}{K_{a}(p)+g^{2} G_{S M}(p)}
$$

where $G_{i}(p)=\left\langle O_{i}(p) O_{i}(-p)\right\rangle_{i}$ is the unperturbed two-point correlation function of $O_{i}$ in theory $i$, in this case the SM. The kinetic function $K_{a}$ can be determined comparing the two-point correlator function of $O_{S M}$ calculated from both actions (3.3) and (3.5) obtaining the following consistency relation

$$
K_{a}(p)=-\frac{\lambda_{0}^{2} M^{2 \Delta_{h}}}{G_{h}(p)}
$$




\subsection{Analysis of the possible IR expansions}

The next step is to investigate the IR expansions of the two-point correlation functions $G_{h}$ and $G_{S M}$, they depend crucially on the detailed physics of each theory. In a theory with a single scale, like a mass gap $m$ in Yang-Mills (YM) theories, the IR expansion in $p \ll m$ reads

$$
i\langle O(p) O(-p)\rangle \simeq A_{0}+A_{2} p^{2}+A_{4} p^{4}+\cdots \quad, \quad A_{n} \sim m^{2(\Delta-2)-n}
$$

where $\Delta$ is the dimension of the operator $O$. If $\Delta$ is an integer, then starting with the term $p^{2(\Delta-2)}$ $\operatorname{logs}$ of momentum appear in the expansion. In the UV regime $p \gg m$ the expansion of the (renormalized) correlator is

$$
i\langle O(p) O(-p)\rangle=p^{2(\Delta-2)}\left\{\log \frac{p^{2}}{m^{2}}\left[B_{0}+B_{2} \frac{m^{2}}{p^{2}}+\mathscr{O}\left(\frac{m^{4}}{p^{4}}\right)\right]+C_{0}+C_{2} \frac{m^{2}}{p^{2}}+\mathscr{O}\left(\frac{m^{4}}{p^{4}}\right)\right\}
$$

where the coefficients $B_{i}, C_{i}$ are dimensionless. The expansion (3.10) is valid whether $\Delta$ is integer or not. If the theory has an UV scale $\Lambda$, together with smaller IR scales, then for generic scalar operators the larger scale dominates the coefficients in the IR expansion (3.9),

$$
A_{n} \sim \Lambda^{2(\Delta-2)-n}\left[1+\mathscr{O}\left(\frac{m^{2}}{\Lambda^{2}}\right)\right]
$$

We now specialize the above analysis to the instanton density operator. It is well known from studies in QFT, [75], and holography, [76], that the correlators of the instanton density are $U V$ insensitive. The reason is that the $\theta$ angle in QCD is not renormalized ${ }^{7}$. This is a key feature that distinguishes the instanton density operator from other scalar operators. Therefore, the two-point function scales as in (3.9) where $m$ is the characteristic IR scale of the gauge theory and the UV scale $\Lambda$ does not appear in the correlator ${ }^{8}$.

The case of a CFT must be discussed separately. In an exact CFT, the two-point function of an appropriately normalized scalar operator in configuration space is $\langle O(x) O(0)\rangle=|x|^{-2 \Delta}$. The Fourier transform is ill-defined for $\Delta>2$ in four dimensions and a regularisation is needed. Using a short-distance cutoff $\Lambda$ one obtains

$$
\langle O(p) O(-p)\rangle \sim p^{2(\Delta-2)}\left(\frac{p}{\Lambda}\right)^{2-\Delta} K_{\Delta-2}\left(\frac{p}{\Lambda}\right)
$$

valid for $p \ll \Lambda$. For $\Delta>2$ it exhibits a logarithmic UV divergence associated with the Bessel $\mathrm{K}$-function.

If there is a non-trivial CFT in the IR, then there are also non-analytic contributions. For example, for an operator with IR dimension $\Delta_{I R}$ we have instead

$$
i\langle O(p) O(-p)\rangle \simeq A_{0}+A_{2} p^{2}+A_{4} p^{4}+\cdots+p^{2\left(\Delta_{I R}-2\right)} \log p^{2}\left(A_{0}^{1}+A_{2}^{1} p^{2}+\cdots\right)+\cdots
$$

\footnotetext{
${ }^{7}$ This was shown rigorously on the lattice [76]-[79]. This is also true in holography, [80, 81]. However in holographic QCD there is a non-trivial $\beta$-function for $\theta$, [76]. Despite this, all $\theta$-dependent contributions to the vacuum energy are cutoff independent.

${ }^{8}$ See also [82].
} 


\subsection{The IR structure}

To study the physical properties of the field we integrated in, we now consider several regimes given by different IR behaviours of two-point functions $G_{h}$ and $G_{S M}$. We will denote by $m_{h}$ and $m_{S M}$ the mass gaps of the hidden theory and the SM respectively. $m_{S M}$ should also be identified with $\Lambda_{Q C D}$. The phenomenologically interesting case is $M \gg m_{S M} \gg m_{h}$, but we will study also other regimes.

The regime $p \ll m_{h}, m_{S M} \ll M$. In this regime we have $p \ll \min \left(m_{h}, m_{S M}\right)$, therefore the IR behavior of the correlators $G_{h}$ and $G_{S M}$ is as follow

$$
i G_{h}(p)=a_{0}+a_{2} p^{2}+a_{4} p^{4}+\cdots \quad, \quad i G_{S M}(p)=b_{0}+b_{2} p^{2}+b_{4} p^{4}+\cdots
$$

Using (3.7), (3.8) and $g \sim M^{4-\Delta_{S M}}$ we can extract from the IR expansion the axion data $m_{a}$ and $f_{a}$, which reads

$$
f_{a}^{2}=\frac{a_{2}}{a_{0}^{2} \lambda_{0}^{2}} M^{2 \Delta_{h}}-b_{2} M^{8-2 \Delta_{S M}} \quad, \quad m_{a}^{2}=\frac{a_{0}}{a_{2}}+\frac{a_{0}^{2}}{a_{2}^{2}}\left(a_{2} b_{0}+a_{0} b_{2}\right) M^{8-2 \Delta_{S M}-2 \Delta_{h}}
$$

For generic scalar operators, we have $a_{n} \sim M^{2\left(\Delta_{h}-2\right)-n}$ and $b_{n} \sim M^{2\left(\Delta_{h}-2\right)-n}$, therefore the axion parameters read

$$
m_{a}^{2} \sim M^{2}, f_{a}^{2} \sim \frac{M^{2}}{\lambda_{0}^{2}}
$$

The induced interaction is weak however general scalar operators acquire large masses.

On the other hand, if the operators $O_{h}$ and $O_{S M}$ are the instanton densities, then their two-point functions are UV insensitive and in this case

$$
a_{n}=\bar{a}_{n} m_{h}^{4-n} \quad, \quad b_{n}=\bar{b}_{n} m_{S M}^{4-n}
$$

where $\bar{a}_{n}, \bar{b}_{n}$ are dimensionless and typically $\mathscr{O}(1)$ coefficients. For instanton densities $\Delta$ is integer, therefore the low energy structure of the two-point functions is of the form presented in (3.13). They present a non-analytic term, however it appears at fourth order in derivatives and does not effect $m_{a}$ and $f_{a}$. In this special case we obtain

$$
f_{a}^{2}=\frac{\bar{a}_{2}}{\bar{a}_{0}^{2}} \frac{M^{8}}{\lambda_{0}^{2} m_{h}^{6}}-\bar{b}_{2} m_{S M}^{2} \quad, \quad m_{a}^{2}=\frac{\bar{a}_{0}}{\bar{a}_{2}} m_{h}^{2}+\frac{\bar{a}_{0}^{2}}{\bar{a}_{2}^{2}} \frac{\lambda_{0}^{2} m_{h}^{6}}{M^{8}} m_{S M}^{2}\left(m_{S M}^{2} \bar{a}_{2} \bar{b}_{0}+m_{h}^{2} \bar{a}_{0} \bar{b}_{2}\right)
$$

In the present regime, $m_{h}, m_{S M} \ll M$, SM corrections (terms involving the coefficients $\bar{b}_{n}$ ) are suppressed by the messenger scale $M$, therefore the axion data are substantially determined by the hidden theory

$$
f_{a}^{2} \sim \frac{1}{\lambda_{0}^{2}} \frac{M^{8}}{m_{h}^{6}}, m_{a}^{2} \sim m_{h}^{2}
$$

Since $m_{h} \ll M$, this is an emergent weakly-coupled axion-like field. If $m_{h}$ is comparable or smaller than SM scales, then this resembles a standard PQ axion. It has however a compositeness scale that affects its low energy properties. 
Despite these similarities with standard PQ axion, the emergent axion has a different origin. It is not related to a continuous global symmetry of a QFT at a higher scale, but an (approximate) "emergent PQ symmetry" arising from a hidden instanton density9.

As shown in (3.19), in our setup the axion mass is mostly $m_{h}$, therefore the phenomenologically interesting case is the limit $m_{h} \ll m_{S M}$. Coming back to axion data with SM corrections in equation (3.18), with the assumption $m_{h} \ll m_{S M}$, we obtain

$$
f_{a}^{2} \simeq \frac{M^{8}}{m_{h}^{6}}+m_{S M}^{2} \quad, \quad m_{a}^{2} \simeq m_{h}^{2}+\frac{m_{S M}^{4}}{f_{a}^{2}}
$$

where we have neglected $\mathscr{O}(1)$ coefficients. The correction to $f_{a}$ is induced by perturbative loop corrections, while non-perturbative QCD effects provide a mass correction of order $\Lambda_{Q C D}^{2} / f_{a}$. The EM instanton density is not expected to contribute to the axion mass.

We should notice that the above calculations can change with the inclusion of cubic and quartic couplings in (3.3). However, as carefully shown in [1] in a perturbative setup, in the case of instanton densities these extra interactions do not spoil the UV insensitivity of the two-point correlator functions.

The regime $m_{h} \gg p \gg m_{S M}$. In this regime the $0^{+-}$glueball of the hidden theory is point-like and featureless while the associated QCD glueball is fat and unstable. This is precisely the behaviour of a fundamental axion field. The hidden two-point function has still the form (3.14) while the SM two-point function is instead

$$
i G_{S M}=p^{4} \log \frac{p^{2}}{m_{S M}^{2}}\left[\hat{b}_{0}+\hat{b}_{2} \frac{m_{S M}^{2}}{p^{2}}+\mathscr{O}\left(\frac{m_{S M}^{4}}{p^{4}}\right)\right] \quad, \quad p \gg m_{S M}
$$

In this case, $\lambda^{2} G_{S M} \ll G_{h}^{-1}$ and the SM corrections to the axion kinetic data are tiny. Therefore, in this regime the axion data are given by (3.19) and are determined by the scales of the hidden theory as well as the messenger scale.

The regime $m_{S M} \gg p \gg m_{h}$. Here the $0^{+-}$glueball of the hidden theory is fat while the associated QCD glueball is point-like and unstable. This is clearly distinct from the behavior of a fundamental axion field. The SM two-point function is given again by (3.14) but now the hidden two-point function becomes

$$
i G_{h}(p)=p^{4} \log \frac{p^{2}}{m_{h}^{2}}\left[-\hat{a}_{0}+\hat{a}_{2} \frac{m_{h}^{2}}{p^{2}}+\mathscr{O}\left(\frac{m_{h}^{4}}{p^{4}}\right)\right], p \gg m_{h}
$$

with $\hat{a}_{0}, \hat{a}_{2}$ dimensionless numbers of order $\mathscr{O}(1)$. In this regime $G_{h}^{-1}$ dominates the $a$ propagator and we obtain

$$
\langle a a\rangle^{-1} \sim \frac{M^{8}}{p^{4} \log \frac{p^{2}}{m_{h}^{2}}}
$$

\footnotetext{
${ }^{9}$ It is expected that all global symmetries are broken by quantum gravitational effects. This in the past has raised the question of the quality of the PQ axion which has been discussed in [83].
} 
This is a non-standard non-local axion kinetic term that in configuration space behaves as $\langle a a\rangle^{-1} \sim$ $M^{8} \log |x|$ and in configuration space the quadratic term for the axion is

$$
S_{\text {eff }} \simeq \frac{M^{8}}{2} \int d^{4} x_{1} d^{4} x_{2} a\left(x_{1}\right) \log \frac{\left|x_{1}-x_{2}\right|}{m_{h}} a\left(x_{2}\right)+\int d^{4} x a(x) O_{S M}(x)
$$

up to factors of order $\mathscr{O}(1)$. It should be stressed that such an unusual (highly non-local) quadratic term is valid for distances $m_{S M}^{-1}<\ell<m_{h}^{-1}$. Such axions are still interesting and well-defined, however the experimental viability is different and standard experimental constraints do not directly apply.

CFT hidden theory. This is a particular case of the previous regime $m_{S M} \gg p \gg m_{h}$ in which $m_{h} \rightarrow 0$. In that case, the only non-trivial scale is the messenger scale that breaks scale invariance in the UV and in such a case the $\left\langle O_{h} O_{h}\right\rangle$ correlator starts in the IR as $p^{4} \log p^{2}$. Therefore the emergent semiclassical axion is "massless" and non-local. This is reminiscent of the Witten-Weinberg theorem, [84], although this theorem applies to emergent massless gravitons and photons.

The regime $M \gg p \gg \max \left(m_{h}, m_{S M}\right)$. In this regime both glueballs are fat and the couplings are expected to be non-local. Indeed, both $G_{h}$ and $G_{S M}$ are given by (3.22) and (3.21), therefore we obtain

$$
\langle a a\rangle^{-1} \sim \frac{M^{8}}{N^{2} \lambda_{0}^{2} p^{4} \log \frac{p^{2}}{m_{h}^{2}}}
$$

In this regime, the axion kinetic term is similar to the one shown in equation (3.24).

\section{The holographic axion}

We now investigate the special case where the hidden theory is a large- $N$ holographic theory. The action $S_{h}$ in equation (3.3) is the action of the holographic theory. Applying the holographic correspondence, we can write ${ }^{10}$

$$
\left\langle e^{i S_{\mathrm{int}}}\right\rangle_{h}=\int_{\lim _{z \rightarrow 0} a(x, z)=O_{S M}(x)} \mathscr{D} a e^{i S_{\text {bulk }}[a]}
$$

where on the left, the expectation value is taken in the hidden holographic theory. $S_{\text {bulk }}[a]$ is the bulk gravity action, $z$ is the holographic coordinate, $a$ is the bulk field dual to the operator $O_{h}$ of dimension $\Delta=4$ and the gravitational path integral has boundary conditions for $a$ to asymptote to the operator $O_{S M}$ near the AdS boundary. We have also neglected the other bulk fields.

Performing a Legendre transform on (4.1) the Schwinger functional of the bulk axion which becomes the bulk effective action

$$
\left\langle e^{i S_{\mathrm{int}}}\right\rangle_{h}=\int_{\lim _{z \rightarrow 0} \partial_{z} a(x, z)=z^{3} k(x)} \mathscr{D} a(x, z) \mathscr{D} k(x) e^{i S_{N}[a]-i \int k(x) O_{S M}(x)}
$$

\footnotetext{
${ }^{10}$ For a scalar operator $O_{\Delta}(x)$ of dimension $\Delta$ dual to a field $\Phi_{M}(x, z)$ of mass $(M \ell)^{2}=\Delta(\Delta-4)$ the asymptotic behaviour would be $\Phi_{M}(x, z) \approx z^{\Delta-4} O_{\Delta}(x)$.
} 
where $k(x)$ is the expectation value of the operator $O_{h}$. We may imagine the SM action as coupled at the radial scale $z_{0} \sim 1 / M$ to the bulk action. Following holographic renormalization [80, 81], we may then rewrite the full bulk+brane action of the emergent axion as $S_{\text {tot }}=S_{\text {bulk }}+S_{\text {brane }}$ where

$$
\begin{gathered}
S_{\text {bulk }}=M_{P}^{3} \int d^{5} x \sqrt{g}\left[Z(\partial a)^{2}+\mathscr{O}\left((\partial a)^{4}\right)\right] \\
S_{\text {brane }}=\delta\left(z-z_{0}\right) \int d^{4} x \sqrt{\gamma}\left[\lambda \hat{a}(x) O_{S M}(x)+M^{2}(\partial \hat{a})^{2}-\Lambda^{4} \hat{a}^{2}+\cdots\right]
\end{gathered}
$$

In the brane action, $\hat{a}(x) \equiv a\left(z_{0}, x\right)$ is the induced axion on the brane and $\gamma$ is the induced fourdimensional metric. As we will be interested at energies $E \ll M$ we can ignore higher derivative terms. In the bulk action, we have also neglected the graviton and other scalar fields dual to other scalar operators.

The first term in (4.4) is the coupling of the axion to the SM instanton densities. The kinetic and mass terms of the axion in the brane action come from the quantum effects of the SM fields. The factor $Z$ in the bulk action in general depends on the various other scalars fields.

For the case of holographic YM this action has been studied in detail in [76, 85]. Importantly, there is a bulk potential for the axion but it is due to instantons and therefore it is exponentially suppressed at large $N$, therefore it can be neglected. To all orders in $1 / N$, the bulk axion has only derivative interactions. It should be noted that what we have here is a close analogue of the DGP mechanism, [86]-[88], with two differences: here we have an axion and also the bulk data are non-trivial.

The main difference in the physics of an emergent axion originating in a holographic theory is that, due to the strong coupling effects, there is an infinity of axion-like resonances coupled to the SM instanton densities. They correspond to the poles of the two-point function of the instanton density of the hidden holographic theory. If the holographic theory is gapless, then there is a continuum of modes and the induced axionic interaction is non-local. If the theory has a gap as large- $N$ YM then there is a tower of nearly stable states at large $N$ that are essentially the $0^{+-}$ glueball trajectory and act as the KK modes of the bulk axion that couple with variable strengths to the SM instanton densities.

To investigate these interactions we analyze the propagator of the axion on the SM brane. For more details see [1] and also [88]-[93]. The axion propagator can be written in terms of the bulk propagator $G_{0}$ with Neumann boundary conditions at the boundary as follow

$$
G\left(p, z ; z_{0}\right)=\frac{G_{0}\left(p, z ; z_{0}\right)}{1+\left(M^{2} p^{2}+\Lambda^{4}\right) G_{0}\left(p, z_{0} ; z_{0}\right)}
$$

The propagator on the brane is obtained by setting $z=z_{0}$. The general structure of the bulk axion propagator $G_{0}$ is known, [88] and is as follows. The position of the brane $z_{0}$ determines a bulk curvature energy scale, $R_{0}$. Assuming that the theory does not have multiple intermediate physics scales we obtain

$$
G_{0}\left(p, z_{0} ; z_{0}\right)=\frac{1}{2 M_{P}^{3}} \begin{cases}p^{-1}, & p \gg R_{0} \\ d_{0}-d_{2} p^{2}-d_{4} p^{4}+\cdots, & p \ll R_{0} .\end{cases}
$$

where $d_{n}=\bar{d}_{n} \ell^{-3} m^{-4-n} . m$ is the characteristic scale of the (hidden) dual QFT, $\ell$ is the IR AdS length and $\bar{d}_{n}$ are dimensionless numbers of order $\mathscr{O}(1)$ and $\left(M_{P} \ell\right)^{3} \sim N^{2}$. The IR expansion above 
is valid for all holographic RG flows. It starts having non-analytic terms at order $p^{4} \log p^{2}$ as is the case with the bulk axion field, [88].

Using (4.6) we now investigate the axion interaction on the SM brane from $G\left(p, z_{0} ; z_{0}\right)$ in (4.5). At short enough distances, $p \rightarrow \infty$, the axion propagator becomes

$$
G_{0}\left(p, z_{0} ; z_{0}\right) \simeq \frac{1}{M^{2}} \frac{1}{p^{2}}
$$

which is the propagator of a massless four-dimensional scalar. For sufficiently small momenta, $p \ll m$, we obtain instead

$$
G^{-1}\left(p, z_{0} ; z_{0}\right) \simeq \Lambda^{4}+2 \frac{M_{P}^{3} \ell^{3} m^{4}}{\bar{d}_{0}}+\left(M^{2}-2 M_{P}^{3} \ell^{3} m^{2} \frac{\bar{d}_{2}}{\bar{d}_{0}^{2}}\right) p^{2}+\frac{2 M_{P}^{3} \ell^{3}}{\bar{d}_{0}}\left[\frac{\bar{d}_{2}^{2}}{\bar{d}_{0}^{2}}+\frac{\bar{d}_{4}}{\bar{d}_{0}}\right] p^{4}+\mathscr{O}\left(p^{6}\right)
$$

We may then recast (4.8) as the propagator of a massive four-dimensional scalar with effective mass and coupling strengths

$$
f_{e f f}^{2}=M^{2}+2 \frac{\bar{d}_{2}}{\bar{d}_{0}^{2}} N^{2} m^{2} \quad, \quad m_{e f f}^{2} f_{e f f}^{2}=\Lambda^{4}+\frac{2}{\bar{d}_{0}} N^{2} m^{4}
$$

Moreover, the coefficient of the $p^{4}$ term is dimensionless and of order $\mathscr{O}\left(N^{2}\right)$. In the limit $N m \gg M$ we obtain $m_{\text {eff }} \sim m$ and $f_{\text {eff }} \sim N m$ which resemble the non-holographic case.

In the regime $M \gg p \gg m$, depending on the hierarchy of the various scales of the problem at intermediate distances, it may be that $\left(M^{2} p^{2}+\Lambda^{4}\right) G_{0}\left(p, z_{0} ; z_{0}\right) \ll 1$ and the axion may behave as a 5-dimensional massless scalar with propagator

$$
G_{0}\left(p, z_{0} ; z_{0}\right) \simeq \frac{1}{2 M_{P}^{3}} \frac{1}{p}
$$

In such a regime, all axion resonances contribute equitably and the resumed result is as above.

\section{Phenomenological considerations}

In this section we compare our general setup with phenomenological constraints on the axion data $f_{a}$ and $m_{a}$, [23]. It should be however stressed at this stage, that in many cases this comparison is superficial. The "kinetic" operators for the axions we consider can be highly non-standard in some regimes, while almost all experimental constraints have been derived with standard kinetic terms.

The precise role of axions in inflation as well as that in axion monodromy models, depends on the details of the axion potential, something that is beyond the scope of the present investigation ${ }^{11}$. Relevant couplings, such as $g_{a \gamma}$ (axion-photon), $g_{a \ell}$ (axion-lepton) and $g_{a N}$ (axion-nucleon) ${ }^{12}$, that can be probed in direct detection and/or (local) astrophysical experiments, are model dependent and this is not something we have analyzed here. The emergent axion may also play the role of a relaxion, [88, 96, 97].

The Weak Gravity Conjecture [98]-[100] states that the decay constant $f_{a}$ cannot be larger than the Plank scale

$$
f_{a}<M_{P} \approx 10^{19} \mathrm{GeV}
$$

\footnotetext{
${ }^{11}$ The role of the emergent axion as an inflaton may be investigated along the lines of [94].

${ }^{12}$ See also [95].
} 


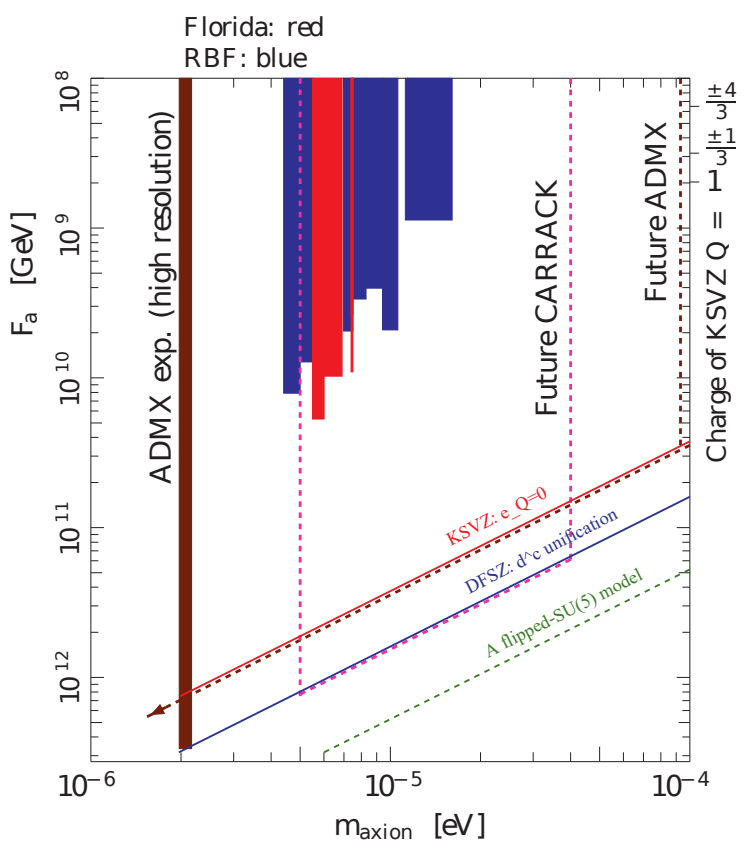

Figure 1: Experimental constraints on the axion parameters, $f_{a}, m_{a}$. Adapted from [20].

Dark matter and dark energy axions. To address dark energy and ultra-light dark matter, the relevant range for ultra-light axions (ULA) is

$$
10^{-33} \mathrm{eV}<m_{a}<10^{-18} \mathrm{eV}
$$

the lower bound being set by $m_{a}>M_{H}{ }^{13}$ (from CMB, axion dark energy) and the upper bound from Baryon Jeans scale (from constraints on large scale structure (LSS) formation and the Epoch of Reionization (EoR)).

The lower end, $10^{-33} \mathrm{eV}<m_{a}<10^{-30} \mathrm{eV}$, corresponds to axions that could account for the present Dark Energy (cosmological constant), the decay constant is required to be $f_{a} \sim M_{P}$. The region $10^{-24} \mathrm{eV}<m_{a}<10^{-18} \mathrm{eV}$ corresponds to axions that could be good candidates for Dark Matter. A typical upper bound on axion density and the decay constant are $\Omega_{a} h^{2}<0.12$ and $f_{a} \leq 10^{16} \mathrm{GeV}$.

The QCD axion. Among the 'historical' QCD axion models, PQWW [6, 101, 102] has been ruled out by experiments, while KSVZ $[10,11]$ (heavy quarks and PQ scalar), as well as DFSZ $[12,13]$ (two Higgses and PQ scalar), are still viable, fig 1. Constraints on QCD axions can be summarized as follow

$$
\begin{aligned}
& 10^{-12} \mathrm{eV}<m_{a}^{Q C D}<10^{-3} \mathrm{eV} \\
& 10^{9} \mathrm{GeV}<f_{a}^{Q C D}<10^{15} \mathrm{GeV}
\end{aligned}
$$

so that $10^{-6} \mathrm{GeV}<\Lambda_{a}^{Q C D}<10^{2} \mathrm{GeV}$, indeed $\Lambda_{a}^{Q C D}=\left(m_{u} \Lambda_{Q C D}^{3}\right)^{1 / 4} \approx 10^{-1} \mathrm{GeV}$. The upper bound on the mass here comes from the one-loop anomaly diagram that correlates $f_{a}$ with $m_{a}$. The

\footnotetext{
${ }^{13} M_{H}$ is defined in terms of today's Hubble-Lemaître constant to be $H\left(t_{0}\right)=H_{0}=100 h(\mathrm{~km} / \mathrm{s}) / \mathrm{Mpc}=$ $2.13 h 10^{-33} \mathrm{eV}=h M_{H}$ with a typical value being $h=0.67$ (or lower).
} 
lower bound for $f_{a}$ comes from supernovae cooling and the upper bound on $f_{a}$ from black hole superradiance arguments.

Heavy axions. There could also exist heavy axions with masses larger than $1 \mathrm{eV}$, [103]. Their mass is constrained in the following two windows

$$
m_{a}>10 \mathrm{MeV} \text { and } \tau_{a \gamma}<10^{-2} \mathrm{~s}, m_{a}<10 \mathrm{eV} \quad \text { or } \tau_{a \gamma}>10^{24} \mathrm{~s}
$$

where $\tau_{a \gamma}$ is the lifetime for decay into photons. ${ }^{14}$

\subsection{Comparison with data}

We now contrast our previous frameworks with the various phenomenological constraints.

Composite Axions. For composite axions in the regime $p \ll m_{h}, m_{S M} \ll M$ we have from (3.19)

$$
m_{a}^{2} \approx m_{h}^{2} \quad, \quad f_{a}^{2} \approx m_{h}^{2}\left(\frac{M}{m_{h}}\right)^{8}
$$

where we are assuming $\bar{a}_{0} / \bar{a}_{2}, \lambda_{0} \approx 1$. The mass $m_{a}$ is completely determined by the hidden mass scale $m_{h}$ and the decay constant $f_{a}$ can be hierarchically larger thanks to the enhancement factor $\left(M / m_{h}\right)^{4}$. The messenger scale is given by

$$
M \approx\left(f_{a} m_{h}^{3}\right)^{1 / 4} \approx\left(f_{a} m_{a}^{3}\right)^{1 / 4}
$$

From the assumption $m_{S M} \ll M$ and the weakest upper bound $f_{a}<M_{P}$, we obtain a general lower bound for the axion mass

$$
m_{a} \gg 10 \mathrm{eV}
$$

Therefore composite axions can be generically compared to heavy axions scenarios only. However, the first window for heavy axions (5.4) is allowed provided the bound on $\tau_{a \gamma}$ is small enough. This can be achieved if $m_{a}$ is sufficiently high, or there is a symmetry reason for suppressing the axion coupling to two photons.

In the case $m_{h}<m_{S M}$, considering again $f_{a}<M_{P}$, the messenger scale has an upper bound

$$
M \lesssim 10 \mathrm{TeV}
$$

which satisfies also $m_{S M} \ll M$. In the case $m_{h}>m_{S M}$ the assumptions are satisfied taking $f_{a} \gg m_{a}$ and the scales $M, m_{a}$ and $f_{a}$ can range from $m_{S M}$ to the Plank scale.

For the QCD axion the messenger scale can be written as $M^{2} \sim m_{a} \Lambda_{a}$, where

$$
\Lambda_{a}=\sqrt{m_{a} f_{a}} \sim\left(m_{u} \Lambda_{Q C D}^{3}\right)^{1 / 4} \sim 10^{-1} \mathrm{GeV} \sim m_{S M}
$$

${ }^{14} \tau_{a \gamma}$ can be computed from the associated decay width as, [104],

$$
\Gamma_{a \rightarrow \gamma \gamma}=\frac{1}{\tau_{a \gamma}}=\frac{G_{a \gamma \gamma}^{2} m_{a}^{3}}{64 \pi}
$$

where $G_{a \gamma \gamma}$ is defined as the coupling of the axion to the EM CP-odd density and it is of order $G_{s \gamma \gamma} \sim \frac{\alpha_{E M}}{f_{a}}$. 
In this case the conditions $m_{h}, m_{S M} \ll M$ cannot be satisfied together.

An obvious issue here is the assumption $p \ll m_{h}$ in the formulae used.

It is not clear whether this holds if $m_{h} \sim 10^{-23} \mathrm{eV}$, for example. If we instead use the formulae $m_{h} \ll p \ll m_{S M}$, the axion is unfortunately non-local and we need a more careful first principles computation to determine the viability of our model.

Holographic Axions. For holographic axions with $p<m_{h} \ll M$ we have from (4.9)

$$
m_{a}^{2} f_{a}^{2}=\Lambda^{4}+\frac{2}{\bar{d}_{0}} N^{2} m_{h}^{4} \quad, \quad f_{a}^{2}=M^{2}+2 \frac{\bar{d}_{2}}{\bar{d}_{0}^{2}} N^{2} m_{h}^{2}
$$

where $\Lambda, M$ are parameters of the brane (visible) theory while $N, m_{h}$ are parameters of the bulk (hidden) theory. Eliminating the combination $N$ from these equations and assuming that $\Lambda^{4}=\kappa \Lambda_{a}^{4}$ (with $\kappa$ of order 1 ), the messenger scale turns out to be

$$
M=f_{a} \sqrt{1+\gamma \kappa \frac{m_{a}^{2}}{m_{h}^{2}}}
$$

where $\gamma=\bar{d}_{2} / \bar{d}_{0}$ is a constant of order 1 . The above relation produces in all cases (QCD, DM and DE) reasonable messenger scales $M \approx f_{a}$ for any $m_{a} \leq m_{h}$. For $m_{a} \gg m_{h}$ we still obtain a reasonable relation $M \approx f_{a}\left(m_{a} / m_{h}\right) \gg f_{a}$. In the regime $m_{h}<p<M$, we obtain an effective 5-dimensional axion.

\section{Results}

In [1] we have shown that axions may arise from large- $N$ strongly-coupled gauge theories as composite instanton densities. These operators have two crucial properties: they possess approximate shift symmetries in the IR and their two-point correlators are UV insensitive.

The first property differentiates these emergent axions from the usual composite axions, which rely on a fermionic PQ symmetry. The second property ensures that their mass can be small and, therefore, they can be visible in the IR. In our setup the axion mass is mostly determined by the characteristic scale of the hidden theory, while the decay constant can be hierarchically higher thanks to the messenger scale.

Comparing our results with data we conclude that generic emergent axions cannot serve like QCD axions, dark matter axions or dark energy axions. They can only play the role of inflatons and heavy axion like particles.

There are cases, when the characteristic scale of the hidden theory is sufficiently low that the axion has a non-local kinetic term. Such axions are still interesting and well-defined, however the analysis of the experimental viability is different, and standard experimental constraints do not directly apply.

A special case is when the hidden theory is a five dimensional holographic theory and the emergent axion is holographic. We find that the interaction at short and long distances is the one of a four-dimensional massive scalar. Depending on some parameters, at intermediate distances there may be a phase where the axion interaction is five-dimensional. Holographic axions, contrary to with generic emergent axions, can play the role of the QCD axion, dark matter and dark energy axions. 


\section{Acknowledgments}

The authors would like to thank the organisers of Corfu Summer Institute 2019 "School and Workshops on Elementary Particle Physics and Gravity" for giving us the opportunity to present our work. This work was supported in part by the Advanced ERC grant SM-grav, No 669288. P.A. and D.C. was supported by FWF Austrian Science Fund via the SAP P30531-N27. This work was partially supported by the MIUR-PRIN contract 2015MP2CX4002 "Nonperturbative aspects of gauge theories and strings" and by the grant "Strong Interactions: from Lattice QCD to Strings, Branes and Holography" (CUP E84I19002260005, CUN Area 02), within the scheme "Beyond Borders" of the University of Roma "Tor Vergata".

\section{References}

[1] P. Anastasopoulos, P. Betzios, M. Bianchi, D. Consoli and E. Kiritsis, "Emergent/Composite axions," JHEP 1910, 113 (2019) [JHEP 2019, 113 (2020)] [ArXiv:1811.05940][hep-ph].

[2] E. Kiritsis, “Gravity and axions from a random UV QFT,” EPJ Web Conf. 71, 00068 (2014) [ArXiv:1408.3541][hep-ph].

[3] M. Baggioli, P. Betzios, E. Kiritsis and V. Niarchos, "Emergent gravity from hidden sectors," to appear.

[4] P. Anastasopoulos, M. Bianchi, D. Consoli and E. Kiritsis, "Graviphotons and dark photons in string theory and QFT," to appear.

[5] P. Betzios, E. Kiritsis and O. Papadoulaki and V. Niarchos, "Emergent U(1)s from hidden sectors," to appear.

[6] R. D. Peccei and H. R. Quinn, “CP Conservation in the Presence of Instantons,” Phys. Rev. Lett. 38 (1977) 1440.

[7] A. Delgado, J. R. Espinosa and M. Quiros, “Unparticles Higgs Interplay,” JHEP 0710 (2007) 094 [ArXiv:0707.4309][hep-ph].

[8] E. Witten, “Current Algebra Theorems for the U(1) Goldstone Boson,” Nucl. Phys. B 156 (1979) 269.

[9] G. Veneziano, “U(1) Without Instantons,” Nucl. Phys. B 159 (1979) 213.

[10] J. E. Kim, “Weak Interaction Singlet and Strong CP Invariance,” Phys. Rev. Lett. 43 (1979) 103.

[11] M. A. Shifman, A. I. Vainshtein and V. I. Zakharov, "Can Confinement Ensure Natural CP Invariance of Strong Interactions?,” Nucl. Phys. B 166 (1980) 493.

[12] M. Dine, W. Fischler and M. Srednicki, “A Simple Solution to the Strong CP Problem with a Harmless Axion,” Phys. Lett. 104B (1981) 199.

[13] A. R. Zhitnitsky, “On Possible Suppression of the Axion Hadron Interactions. (In Russian),” Sov. J. Nucl. Phys. 31 (1980) 260 [Yad. Fiz. 31 (1980) 497].

[14] D. B. Kaplan, “Opening the Axion Window,” Nucl. Phys. B 260 (1985) 215.

[15] M. Srednicki, “Axion Couplings to Matter. 1. CP Conserving Parts,” Nucl. Phys. B 260 (1985) 689.

[16] H. Georgi, D. B. Kaplan and L. Randall, “Manifesting the Invisible Axion at Low-energies,” Phys. Lett. 169B (1986) 73. 
[17] K. Choi, K. Kang and J. E. Kim, “Effects of $\eta^{\prime}$ in Low-energy Axion Physics,” Phys. Lett. B 181 (1986) 145.

[18] G. Grilli di Cortona, E. Hardy, J. Pardo Vega and G. Villadoro, “The QCD axion, precisely,” JHEP 1601 (2016) 034 [ArXiv:1511.02867] [hep-ph].

[19] P. Di Vecchia, G. Rossi, G. Veneziano and S. Yankielowicz, "Spontaneous CP breaking in QCD and the axion potential: an effective Lagrangian approach,” JHEP 1712 (2017) 104 [ArXiv:1709.00731][hep-th].

[20] J. E. Kim and G. Carosi, “Axions and the Strong CP Problem,” Rev. Mod. Phys. 82 (2010) 557 [ArXiv:0807.3125] [hep-ph].

[21] F. Bigazzi, A. Caddeo, A. L. Cotrone, P. Di Vecchia and A. Marzolla, "The Holographic QCD Axion,” JHEP 1912, 056 (2019) [ArXiv:1906.12117][hep-th].

[22] H. Baer, K. Y. Choi, J. E. Kim and L. Roszkowski, “Dark matter production in the early Universe: beyond the thermal WIMP paradigm,” Phys. Rept. 555 (2015) 1 [ArXiv:1407.0017] [hep-ph].

[23] D. J. E. Marsh, “Axion Cosmology,” Phys. Rept. 643 (2016) 1 [ArXiv:1510.07633][astro-ph.CO].

[24] S. Alekhin et al., "A facility to Search for Hidden Particles at the CERN SPS: the SHiP physics case," Rept. Prog. Phys. 79 (2016) no.12, 124201 [ArXiv:1504.04855][hep-ph].

[25] P. W. Graham, I. G. Irastorza, S. K. Lamoreaux, A. Lindner and K. A. van Bibber, “Experimental Searches for the Axion and Axion-Like Particles,” Ann. Rev. Nucl. Part. Sci. 65 (2015) 485 [ArXiv:1602.00039][hep-ex].

[26] I. G. Irastorza and J. Redondo, "New experimental approaches in the search for axion-like particles," Prog. Part. Nucl. Phys. 102 (2018) 89 [ArXiv:1801.08127][hep-ph].

[27] P. Svrcek and E. Witten, “Axions In String Theory,” JHEP 0606 (2006) 051 [ArXiv:hep-th/0605206].

[28] A. Arvanitaki, S. Dimopoulos, S. Dubovsky, N. Kaloper and J. March-Russell, "String Axiverse," Phys. Rev. D 81 (2010) 123530 [ArXiv:0905.4720][hep-th].

[29] J. Polchinski, "String theory. Vol. 2: Superstring theory and beyond," doi:10.1017/CBO9780511618123

[30] E. Kiritsis, “String theory in a nutshell,” Princeton University Press, Second Edition, 2019

[31] M. Dine, N. Seiberg, X. G. Wen and E. Witten, "Nonperturbative Effects on the String World Sheet," Nucl. Phys. B 278 (1986) 769.

[32] J. Polchinski, “Dirichlet Branes and Ramond-Ramond charges,” Phys. Rev. Lett. 75 (1995) 4724 [ArXiv:hep-th/9510017].

[33] K. Becker, M. Becker and A. Strominger, “Five-branes, membranes and nonperturbative string theory,” Nucl. Phys. B 456 (1995) 130 [ArXiv:hep-th/9507158].

[34] E. Kiritsis, “Duality and instantons in string theory,” [ArXiv:hep-th/9906018].

[35] M. Billo, M. Frau, I. Pesando, F. Fucito, A. Lerda and A. Liccardo, “Classical gauge instantons from open strings,” JHEP 0302 (2003) 045 [ArXiv:hep-th/0211250].

[36] R. Blumenhagen, M. Cvetic, S. Kachru and T. Weigand, "D-Brane Instantons in Type II Orientifolds,” Ann. Rev. Nucl. Part. Sci. 59 (2009) 269 [ArXiv:0902.3251] [hep-th].

[37] M. B. Green and M. Gutperle, “Effects of D instantons,” Nucl. Phys. B 498 (1997) 195 [ArXiv:hep-th/9701093]. 
[38] E. Kiritsis and B. Pioline, “On $R^{4}$ threshold corrections in IIb string theory and $(p, q)$ string instantons,” Nucl. Phys. B 508 (1997) 509 [ArXiv:hep-th/9707018].

[39] M. Bianchi, M. B. Green, S. Kovacs and G. Rossi, “Instantons in supersymmetric Yang-Mills and D instantons in IIB superstring theory,” JHEP 9808 (1998) 013 [ArXiv:hep-th/9807033].

[40] M. Bianchi, S. Kovacs and G. Rossi, “Instantons and Supersymmetry,” Lect. Notes Phys. 737 (2008) 303 [ArXiv:hep-th/0703142].

[41] M. Bianchi and M. Samsonyan, “Notes on unoriented D-brane instantons,” Int. J. Mod. Phys. A 24 (2009) 5737 [ArXiv:0909.2173][hep-th].

[42] M. Bianchi and E. Kiritsis, "Non-perturbative and Flux superpotentials for Type I strings on the Z(3) orbifold,” Nucl. Phys. B 782 (2007) 26 [ArXiv:hep-th/0702015].

[43] M. Bianchi, F. Fucito and J. F. Morales, "D-brane instantons on the T**6 / Z(3) orientifold", JHEP 0707 (2007) 038 [ArXiv:0704.0784][hep-th].

[44] M. Bianchi, F. Fucito and J. F. Morales, "Dynamical supersymmetry breaking from unoriented D-brane instantons,” JHEP 0908 (2009) 040 [ArXiv:0904.2156][hep-th].

[45] A. Addazi and M. Bianchi, "Neutron Majorana mass from exotic instantons, JHEP 1412 (2014) 089 [ArXiv:1407.2897][hep-ph].

[46] M. Bianchi, J. F. Morales and C. Wen, "Instanton corrections to the effective action of $\mathscr{N}=4$ SYM," JHEP 1511 (2015) 006 [ArXiv:1508.00554][hep-th].

[47] J. E. Kim, “A Composite Invisible Axion,” Phys. Rev. D 31 (1985) 1733.

[48] K. Choi and J. E. Kim, “Dynamical Axion,” Phys. Rev. D 32 (1985) 1828.

[49] R. C. Furlong, “Quen's Gambit: Decuplet Quarks And Composite Axions,” Phys. Lett. B 211 (1988) 450.

[50] L. Randall, “Composite axion models and Planck scale physics,” Phys. Lett. B 284 (1992) 77.

[51] E. A. Dudas, "Composite supersymmetric axion - dilaton - dilatino system and the breaking of supersymmetry,” Phys. Rev. D 49 (1994) 1109 [ArXiv:hep-ph/9307294].

[52] C. T. Hill and A. K. Leibovich, "Natural theories of ultralow mass PNGB's: Axions and quintessence,” Phys. Rev. D 66 (2002) 075010 [ArXiv:hep-ph/0205237].

[53] M. Redi and R. Sato, “Composite Accidental Axions,” JHEP 1605 (2016) 104 [ArXiv:1602.05427][hep-ph].

[54] F. Wilczek and G. Moore, "Superheavy Light Quarks and the Strong P, T Problem,” [ArXiv:1601.02937][hep-ph].

[55] K. R. Dienes, E. Dudas and T. Gherghetta, "Invisible axions and large radius compactifications," Phys. Rev. D 62 (2000) 105023 [ArXiv:hep-ph/9912455].

[56] P. Anastasopoulos, T. P. T. Dijkstra, E. Kiritsis and A. N. Schellekens, "Orientifolds, hypercharge embeddings and the Standard Model,” Nucl. Phys. B 759 (2006) 83; [ArXiv:hep-th/0605226].

[57] I. Antoniadis, E. Kiritsis and T. N. Tomaras, “A D-brane alternative to unification,” Phys. Lett. B 486 (2000) 186 [ArXiv:hep-ph/0004214]; Fortsch. Phys. 49 (2001) 573 [ArXiv:hep-th/0111269].

I. Antoniadis and S. Dimopoulos, "Splitting supersymmetry in string theory,” Nucl. Phys. B 715 (2005) 120 [ArXiv:hep-th/0411032]. 
[58] I. Antoniadis, E. Kiritsis and T. Tomaras, “D-brane standard model,” Fortsch. Phys. 49 (2001) 573 [ArXiv:hep-th/0111269].

[59] E. Kiritsis and P. Anastasopoulos, "The Anomalous magnetic moment of the muon in the D-brane realization of the standard model,” JHEP 0205 (2002) 054 [ArXiv:hep-ph/0201295].

[60] E. Kiritsis, "D-branes in standard model building, gravity and cosmology," Fortsch. Phys. 52 (2004) 200 [ Phys. Rept. 421 (2005) 105-190; Erratum ibid 429 (2006) ,121-122] [ArXiv:hep-th/0310001].

[61] C. Coriano, N. Irges and E. Kiritsis, "On the effective theory of low scale orientifold string vacua," Nucl. Phys. B 746 (2006) 77 [ArXiv:hep-ph/0510332].

[62] I. Antoniadis, E. Kiritsis and J. Rizos, “Anomalous U(1)s in type-I superstring vacua,” Nucl. Phys. B 637 (2002) 92 [ArXiv:hep-th/0204153].

[63] P. Anastasopoulos, “4-D anomalous $U(1)$ 's, their masses and their relation to 6-D anomalies,” JHEP 0308 (2003) 005 [ArXiv:hep-th/0306042].

[64] P. Anastasopoulos, M. Bianchi, E. Dudas and E. Kiritsis, “Anomalies, anomalous U(1)'s and generalized Chern-Simons terms,” JHEP 0611 (2006) 057 [ArXiv:hep-th/0605225].

[65] Karplus, Robert and Neuman, Maurice, “The Scattering of Light by Light,” Phys. Rev. 83 (1951) 776-784

[66] G. Mahlon, “One loop multi - photon helicity amplitudes,” Phys. Rev. D 49 (1994) 2197 [ArXiv:hep-ph/9311213].

[67] M. G. Schmidt and C. Schubert, "On the calculation of effective actions by string methods," Phys. Lett. B 318 (1993) 438 [ArXiv:hep-th/9309055].

[68] A. Ritz and R. Delbourgo, "The Low-energy effective Lagrangian for photon interactions in any dimension,” Int. J. Mod. Phys. A 11 (1996) 253 [ArXiv:hep-th/9503160].

[69] H. Elvang and Y. t. Huang, "Scattering Amplitudes,” [ArXiv:1308.1697][hep-th].

[70] M. Bianchi, H. Elvang and D. Z. Freedman, "Generating Tree Amplitudes in N=4 SYM and N=8 SG,” JHEP 0809 (2008) 063 [ArXiv:0805.0757][hep-th].

[71] M. Bianchi and D. Consoli, “Simplifying one-loop amplitudes in superstring theory,” JHEP 1601 (2016) 043 [ArXiv:1508.00421][hep-th].

[72] P. Anastasopoulos, M. Bianchi and D. Consoli, "Yukawas of light stringy states (at D-brane intersections)," PoS CORFU2017 (2018), 055;

P. Anastasopoulos, M. Bianchi and D. Consoli, "Yukawa couplings for light stringy states," PoS EPS-HEP2017 (2017), 538.

[73] P. Anastasopoulos and R. Richter, "Production of light stringy states," JHEP 12 (2014), 059 [ArXiv:1408.4810][hep-th];

P. Anastasopoulos and R. Richter, “Twisted state production,” PoS CORFU2014 (2015), 116;

P. Anastasopoulos, M. Bianchi and R. Richter, “Light stringy states,” JHEP 03 (2012), 068 [ArXiv:1110.5424][hep-th].

[74] S. Marchesani and Y. Stanev, "Higgs decay in two photons," (2013) Master thesis.

[75] E. Vicari and H. Panagopoulos, "Theta dependence of $S U(N)$ gauge theories in the presence of a topological term,” Phys. Rept. 470 (2009) 93 [ArXiv:0803.1593][hep-th]. 
[76] U. Gürsoy and E. Kiritsis, “Exploring improved holographic theories for QCD: Part I,” JHEP 0802 (2008) 032 [ArXiv:0707.1324][hep-th];

U. Gürsoy, E. Kiritsis, F. Nitti, “Exploring improved holographic theories for QCD: Part II,” JHEP 0802, 019 (2008) [ArXiv:0707.1349][hep-th];

U. Gursoy, E. Kiritsis, L. Mazzanti, G. Michalogiorgakis and F. Nitti, “Improved Holographic QCD," Lect. Notes Phys. 828 (2011) 79 [ArXiv:1006.5461][hep-th].

[77] U. Gursoy, E. Kiritsis, L. Mazzanti and F. Nitti, “Improved Holographic Yang-Mills at Finite Temperature: Comparison with Data,” Nucl. Phys. B 820 (2009) 148 [ArXiv:0903.2859][hep-th].

[78] P. Di Vecchia, K. Fabricius, G. C. Rossi and G. Veneziano, "Numerical Checks of the Lattice Definition Independence of Topological Charge Fluctuations,” Phys. Lett. 108B (1982) 323.

[79] P. Di Vecchia, K. Fabricius, G. C. Rossi and G. Veneziano, "Preliminary Evidence for U(1)-A Breaking in QCD from Lattice Calculations,” Nucl. Phys. B 192 (1981) 392.

[80] M. Bianchi, D. Z. Freedman and K. Skenderis, “Holographic renormalization,” Nucl. Phys. B 631 (2002) 159 [ArXiv:hep-th/0112119].

[81] M. Bianchi, D. Z. Freedman and K. Skenderis, “How to go with an RG flow,” JHEP 0108 (2001) 041 [ArXiv:hep-th/0105276].

[82] A. I. Vainshtein, V. I. Zakharov, V. A. Novikov and M. A. Shifman, “ABC's of Instantons,” Sov. Phys. Usp. 25 (1982) 195 [Usp. Fiz. Nauk 136 (1982) 553];

V. A. Novikov, M. A. Shifman, A. I. Vainshtein and V. I. Zakharov, "Calculations in External Fields in Quantum Chromodynamics. Technical Review,” Fortsch. Phys. 32 (1984) 585.

[83] M. Kamionkowski and J. March-Russell, "Planck scale physics and the Peccei-Quinn mechanism," Phys. Lett. B 282 (1992) 137 [ArXiv:hep-th/9202003];

S. M. Barr and D. Seckel, "Planck scale corrections to axion models,” Phys. Rev. D 46 (1992) 539; R. Holman, S. D. H. Hsu, T. W. Kephart, E. W. Kolb, R. Watkins and L. M. Widrow, "Solutions to the strong CP problem in a world with gravity,” Phys. Lett. B 282 (1992) 132 [ArXiv:hep-ph/9203206].

[84] S. Weinberg and E. Witten, "Limits on Massless Particles," Phys. Lett. 96B, 59 (1980).

[85] U. Gursoy, I. Iatrakis, E. Kiritsis, F. Nitti and A. O'Bannon, “The Chern-Simons Diffusion Rate in Improved Holographic QCD,” JHEP 1302 (2013) 119 [ArXiv:1212.3894] [hep-th]

[86] G. R. Dvali, G. Gabadadze and M. Porrati, “4-D gravity on a brane in 5-D Minkowski space,” Phys. Lett. B 485 (2000) 208 [ArXiv:hep-th/0005016].

[87] E. Kiritsis, N. Tetradis and T. N. Tomaras, “Induced gravity on RS branes,” JHEP 0203 (2002) 019 [ArXiv:hep-th/0202037].

[88] C. Charmousis, E. Kiritsis and F. Nitti, “Holographic self-tuning of the cosmological constant,” JHEP 1709 (2017) 031 [ArXiv:1704.05075][hep-th].

[89] M. Bianchi, O. DeWolfe, D. Z. Freedman and K. Pilch, “Anatomy of two holographic renormalization group flows,” JHEP 0101 (2001) 021 [ArXiv:hep-th/0009156].

[90] E. Kiritsis, N. Tetradis and T. N. Tomaras, "Induced brane gravity: Realizations and limitations," JHEP 0108 (2001) 012 [ArXiv:hep-th/0106050].

[91] M. Bianchi and A. Marchetti, "Holographic three point functions: One step beyond the tradition," Nucl. Phys. B 686 (2004) 261 [ArXiv:hep-th/0302019]. 
[92] M. Bianchi, M. Prisco and W. Mueck, “New results on holographic three point functions,” JHEP 0311 (2003) 052 [ArXiv:hep-th/0310129].

[93] M. Jarvinen and E. Kiritsis, “Holographic Models for QCD in the Veneziano Limit,” JHEP 1203 (2012) 002 [ArXiv:1112.1261][hep-ph];

D. Arean, I. Iatrakis, M. Jarvinen and E. Kiritsis, "The discontinuities of conformal transitions and mass spectra of V-QCD,” JHEP 1311 (2013) 068 [ArXiv:1309.2286][hep-ph];

“CP-odd sector and $\theta$ dynamics in holographic QCD,” Phys. Rev. D 96 (2017) no.2, 026001

[ArXiv:1609.08922][hep-ph].

[94] C. Deffayet, “Cosmology on a brane in Minkowski bulk,” Phys. Lett. B 502 (2001) 199 [ArXiv:hep-th/0010186].

[95] F. Bigazzi, A. L. Cotrone, M. Järvinen and E. Kiritsis, "Non-derivative Axionic Couplings to Nucleons at large and small N,” JHEP 2001, 100 (2020) [ArXiv:1906.12132][hep-ph].

[96] P. W. Graham, D. E. Kaplan and S. Rajendran, “Cosmological Relaxation of the Electroweak Scale,” Phys. Rev. Lett. 115 (2015) no.22, 221801 [ArXiv:1504.07551][hep-ph].

[97] Y. Hamada, E. Kiritsis, F. Nitti, L. Wtkowski, “Axion RG flows and the holographic dynamics of instanton densities,” J. Phys. A 52, no. 45, 454003 (2019) [ArXiv:1905.03663][hep-th].

[98] N. Arkani-Hamed, L. Motl, A. Nicolis and C. Vafa, “The String landscape, black holes and gravity as the weakest force,” JHEP 0706 (2007) 060 [ArXiv:hep-th/0601001].

[99] T. Rudelius, “Constraints on Axion Inflation from the Weak Gravity Conjecture,” JCAP 1509 (2015) no.09, 020 [ArXiv:1503.00795][hep-th];

B. Heidenreich, M. Reece and T. Rudelius, “Weak Gravity Strongly Constrains Large-Field Axion Inflation,” JHEP 1512 (2015) 108 [ArXiv:1506.03447][hep-th].

[100] A. Hebecker, P. Henkenjohann and L. T. Witkowski, "What is the Magnetic Weak Gravity Conjecture for Axions?,” Fortsch. Phys. 65 (2017) no.3-4, 1700011 [ArXiv:1701.06553][hep-th].

[101] S. Weinberg, “A New Light Boson?,” Phys. Rev. Lett. 40 (1978) 223.

[102] F. Wilczek, "Problem of Strong $P$ and $T$ Invariance in the Presence of Instantons," Phys. Rev. Lett. 40 (1978) 279.

[103] M. Millea, L. Knox and B. Fields, "New Bounds for Axions and Axion-Like Particles with keV-GeV Masses,” Phys. Rev. D 92, no. 2, 023010 (2015) [ArXiv:1501.04097][astro-ph].

[104] Particle Data Book 2010 